$\begin{array}{cl}\begin{array}{cl}\text { Revue } \\ \text { de } / \text { histoire }\end{array} & \text { Revue de l'histoire des religions } \\ \text { des religions } & \begin{array}{c}4 \mid 2008 \\ \text { Varia }\end{array}\end{array}$

\title{
Martyrs et reliques en Occident
}

Martyrs and Relics in the West

\section{Bernard Bourrit}

\section{OpenEdition}

Journals

Édition électronique

URL : http://journals.openedition.org/rhr/6913

DOI : 10.4000/rhr.6913

ISSN : 2105-2573

\section{Éditeur}

Armand Colin

\section{Édition imprimée}

Date de publication : 1 décembre 2008

Pagination : 443-470

ISSN : 0035-1423

\section{Référence électronique}

Bernard Bourrit, « Martyrs et reliques en Occident », Revue de l'histoire des religions [En ligne], 4 | 2008,

mis en ligne le 01 octobre 2011, consulté le 30 avril 2019. URL : http://journals.openedition.org/

rhr/6913; DOl : 10.4000/rhr.6913 


\section{Martyrs et reliques en Occident}

Cet essai, consacré aux martyrs et à leurs reliques, entend faire la lumière sur la difficile question de leur invention. J'y soutiens l'idée que le martyr et la vénération rendue à ses restes sont des positions qui s'inventent à la faveur du jeu des forces sociales en présence. J'indique également comment la relique, au sens où on l'entend conventionnellement, ne doit rien à la «ferveur » ou à la « dévotion populaire » et pourquoi son fonctionnement ne se laisse pas décrire en termes de "participation» ou de "métonymie». Mon propos ne se résume donc pas à décrire l'évolution d'un processus historique, mais voudrait problématiser une rupture qui n'a pas encore trouvé de justification.

\section{Martyrs and Relics in the West}

This essay, which is devoted to martyrs and their relics, seeks to clarify the difficult question of their invention. On this subject, I defend the idea that the martyrs and the veneration given to their remains are positions invented by the play of social forces. I also indicate how the relic, conventionally understood, owes nothing to "fervor", nor to "popular devotion", and why its functioning cannot be described in terms of "participation" or "metonymy". My article does not just describe the evolution of a historical process, but it questions a rupture which has yet to be explained. 
Dans le monde occidental, la relique est indissociable du martyr dont elle prolonge l'existence. Sur quel mode la prolonge-t-elle ? Pour le comprendre, il faut distinguer le corps du saint, de l'homme qui vit en état de sainteté. Le saint n'est pas son corps, qu'il cède volontiers aux hommes et à leur justice, mais le réceptacle de la sainteté. À l'image d'une gaine trop lourde, empesée de matière, le saint se débarrasse du corps pour gagner l'au-delà. Mais, à l'inverse, son corps est saint et, s'il arrive qu'on le découpe pour se le partager, chacune de ses parties doit l'être aussi. Chaque parcelle du saint conserve la sainteté du saint. Il y a entre le fragment et la totalité à laquelle il réfère, un rapport de participation. «Le corps a beau être divisé, la grâce demeure entière, proclament les théologiens, et ce tout petit morceau de relique a une puissance égale à celle qu' aurait le martyr si on ne l'avait absolument jamais partagé » ${ }^{1}$. Pourtant, ce disant, rien n'a été dit. Rien d'autre que ce que l'Église énonçait déjà du temps des confesseurs. Or, en abordant la question par le biais d'outils forgés par l'Église elle-même, le risque est grand d'occulter les voix des petits croyants, nouveaux convertis en quête de sens, indécis ou profiteurs sans vergogne, car le point de vue dominant de l'Église n'envisage pas les conditions matérielles dans lesquelles le martyre, et les comportements qui lui sont liés, sont apparus. Dans une telle perspective, nul n'interroge ce que c'est que de céder son corps à la justice des hommes, ce que c'est que découper un corps pour se le partager, ce que $c^{\prime}$ est au juste qu'un martyr.

\section{JUSTICE ET MARTYRE}

Un demi-siècle s'est écoulé depuis le grand incendie de Rome (64), sept empereurs se sont succédé et, si le temps n'est pas encore aux persécutions systématiques de grande ampleur, la délation, la poursuite, l'humiliation et l'exécution de ceux « que la foule appelait "chrétiens" $»^{2}$ ont déjà intégré les affaires courantes de l'Empire.

1. Théodoret de Cyr, Thérapeutique des maladies helléniques, trad. Pierre Canivet, Paris, Le Cerf, 2001, VIII, 11.

2. Tacite, Annales, trad. Pierre Wuilleumier, Paris, Les Belles Lettres, 2003, XV, 44, 2-5. 
Mais à ce stade de la répression, la justice romaine n'engendre pas encore de «martyrs » à proprement parler : la communauté des croyants n'étant pas directement inquiétée, le chrétien qui se voit condamné fait encore figure d'exception. D'ailleurs, la relative clémence, la flexibilité dont bénéficient les adeptes du christianisme sous le règne des Antonins (96-180) s'explique par le caractère flottant de la législation sur les impies sur laquelle l'autorité s'appuie pour réglementer « l'athéisme » chrétien ${ }^{3}$. Car la grande liberté d'interprétation laissée au juge était elle-même inscrite au cœur de cet article de loi : il était prévu en effet que « le proconsul d[evait] établir avec plus ou moins de sévérité ou de clémence la peine d'un sacrilège selon la qualité de la personne et selon la condition de l'accusé en vertu des circonstances, de l'âge et du sexe. » ${ }^{4}$ Arbitrer avec pondération, mesurer la peine, sanctionner l'impiété outrageante : l'activité judiciaire du procurateur de province se borne à régler les litiges. Elle n'est pas l'expression d'une justice tyrannique, mais le reflet d'une politique générale sécuritaire garante de l'état de droit dans l'Empire. L'État, à travers les mesures antichrétiennes qu'il promulgue, tend avant tout à préserver la concorde sociale et la paix civile. C'est que la montée en puissance de la discrimination antichrétienne n'émane ni du pouvoir, ni de l'État, mais procède d'en bas, de la haine ordinaire et des querelles de voisinage, inspirées par la jalousie ou l'envie et alimentées par les dénonciations. Dans ce contexte, il faut insister sur le rôle qu'exerce l'opinion dans la stigmatisation du délit d'athéisme : « Moi aussi, explique Justin (100-165), je m'attends à faire l'objet d'une dénonciation et à être attaché au bois du supplice par l'un de ceux que j'ai nommés [...] amis du bruit et de la parade. Car il n'est pas juste d'appeler "philosophe", un homme qui, parlant à propos de nous, de ce qu'il ne connaît pas, accuse en public les chrétiens d'athéisme et d'impiété, et agit ainsi pour la faveur et le plaisir de la multitude qui est dans l'erreur. $»^{5}$

3. La correspondance entre Pline et Trajan est éloquente sur le sujet. Cf. Pline le Jeune, Lettres, trad. Marcel Durry, Paris, Les Belles Lettres, 2002, X, 96.

4. Ulpien, Digeste, XLVIII, 13, 7, 6. (Traduction personnelle)

5. Justin, Apologie pour les chrétiens, trad. André Wartelle, Paris, Études augustiniennes, 1987, II, 3. 
L'ami du bruit, face à « ce qu'il ne connaît pas », colporte la médisance. À la fois par enflure et souci de plaire, il aime à calomnier son entourage. Mais ses attaques réglées sont grosses de préjugés : « ils sont sots, stupides, insensés et grossiers ces gens qui se raillent et se moquent des [chrétiens], et qui veulent s'enfler de leurs propres pensées. ${ }^{6}$ Le combat d'intelligence semble inégal entre les tenants éclairés de la nouvelle doctrine et leurs adversaires. D'ailleurs combattent-ils seulement les idées, le dogme ou la congrégation des sectateurs ? Non. Ils ne tâchent que de résister à l'effet vicariant de leur doctrine. Les motifs de se plaindre sont terre à terre, leurs traits mal ajustés. Il n'est pas exceptionnel de voir au cours du procès, une fois la lecture du réquisitoire achevée, le juge interpellé en ces termes : "Ce sont là tes arguments ? C'est toute ta science ? Socrate a reçu moins d'outrages de la part des Athéniens! ${ }^{7}$ Quoique la justice suive son cours, le dépositaire de l'autorité a souvent maille à partir avec la finesse de l'accusé qui, moins que l'accusation, conteste la légitimité de l'accusateur. De même que la sanction n'atteignait pas l'élément sanctionné, on constate que le condamné récuse sa condamnation. La compétence du tribunal est écornée par la résistance opiniâtre des confesseurs qui défendent pied à pied leur légitimité. La justice, qui fonctionne pourtant, ne remplit pas sa fonction. Juges et condamnés ne s'articulent pas autour de la sentence comme autour d'une appartenance commune. Issu de la reconnaissance de valeurs de base et de l'acceptation partagée du jeu social, ce sentiment d'appartenance est mis à mal : les faits d'imputation sont systématiquement requalifiés, les jugements disqualifiés.

On verra plus tard comment, reversant la vapeur, la machine judiciaire parviendra à relancer son efficacité et son rendement, mais il convient au préalable de recadrer l'incompétence des tribunaux de province dans son contexte politique en insistant sur le fait que les premières informations contre les chrétiens n'ont pas été ouvertes

6. Clément de Rome, Épître aux Corinthiens, trad. Annie Jaubert, Paris, Le Cerf, 2000, XXXIX, 1-2.

7. « Pionius » dans Les Actes des martyrs. Les premiers martyrs de l'Église \& Les martyrs de la grande persécution, trad. A. G. Hamman, Paris, Desclée de Brouwer, 1979, p. 100. 
sur des décisions émanant du sommet de l'État, mais déclenchées au coup par coup par le harcèlement de la base. Et, cette base, ce n'est ni le zèle procédurier, ni le goût de la dialectique qui l'a décidée à pratiquer la délation, mais la seule concupiscence. Les voix des opprimés sont unanimes à dénoncer cette discrimination aveugle, nourrie par des ressentiments personnels et étayée sur la seule présomption du délateur. «C'est à cause de la jalousie et de l'envie qu'ont été persécutés les [chrétiens] les plus élevés et les plus justes $»^{8}$. La jalousie, en cette acception, est la manifestation d'un ressentiment à peine voilé qui a pour objet le mode de vie du chrétien en général et son ascétisme en particulier. Le païen prend plaisir à outrager, parce qu'il le jalouse, l'homme qui vit en état de perfection. Jalousie et détestation ne sont jamais que l'avers et le revers d'une même médaille. Mais au juste que jalouse le païen ? Qu'y a-t-il de si enviable, et partant de si détestable, dans cette vie tissée d'abstinence, d'ordonnance et de renoncement? Leur ascétisme serait-il criminel ? On n'est pas loin de le croire. C'est bien « en raison de leur haine pour le genre humain $»^{9}$ qu' on dénonçait, recherchait et poursuivait les chrétiens. Ce qui ne doit pas s'entendre en ce sens que les poursuites auraient été engagées à cause du danger qu'ils constituaient pour la société, mais en raison du mépris et de l'indifférence qu'ils affichaient ouvertement pour leur propre personne. Ascétisme criminel, car le fanatique, qui méprise sa mort, méprise aussi en l'espèce l'humanité. Et cette attitude n'aurait pas suscité l'émotion qu'elle a soulevée, si elle ne confinait pas proprement dit à la folie. Il n'est que la démence, note Épictète, la folie furieuse qui puisse donner une idée de l'indifférence avec laquelle les Galiléens ont l'habitude de traiter leur corps ${ }^{10}$. C'est cette haine de soi, inconcevable pour le philosophe et inadmissible aux yeux de la population, qui déchaîne l'indignation. Jalousie des juges et de la foule face à la détermination, à la résistance et à la puissance de volonté des martyrs, jalousie des juges surtout qui « sont torturés

8. Clément de Rome, Épître aux Corinthiens..., V, 2.

9. Tacite, Annales, trad. Pierre Wuilleumier, Paris, Les Belles Lettres, 2003, $\mathrm{XV}, 44,2-5$.

10. Cf. Epictète, Entretien, IV, 7, 6. 
par la patience des chrétiens au milieu des mauvais traitements et des épreuves mais s'enorgueillissent de leur défaite. » ${ }^{11}$ Ainsi prise au piège de la rhétorique chrétienne, l'autorité garante du droit cherche avant tout à éviter de devenir le bras qui favorise l'exécution des attentes millénaristes. Cet expédient nuirait à la régularité de ses jugements. Il est clair que le simulacre de suicide auquel, en se laissant condamner, se livrent les chrétiens devait à court terme discréditer la justesse de la justice ${ }^{12}$. La sanction édifiante qui couronne l'instruction, les sévices terrifiants, la visibilité en milieu urbain, de même que la scénographie qui encadre le corps délictueux, jouent ici en réalité un rôle à contre-emploi. Au lieu que la répression serve d'arme de dissuasion, elle donne vitrine aux doléances des victimes. La signification pénale du procès est détournée au profit de sa portée exemplaire. Pariant que ce gain de visibilité ne diminuera pas le pouvoir de l'Église mais au contraire le renforcera, le chrétien mise sur la spectacularisation de la machine judiciaire. Quelque sévère qu'elle soit, l'issue du procès n'est pas à la hauteur du résultat escompté : elle fabrique du miracle, elle stupéfie le chaland, produit des conversions parmi les badauds. La résistance du martyr force l'admiration générale au détriment de la justice pour finalement témoigner en faveur du nom qu'il confesse. L'impact «médiatique » du procès oblitère les tenants et aboutissants de l'instruction. La persévérance opiniâtre que le martyr dans l'arène met à professer sa foi emprunte au théâtre son décorum, son public, sa popularité et sa renommée. Elle coudoie avec les moyens de la scène, à la limite de la représentation. Dans l'amphithéâtre où, suivant Origène, païens et juifs se réunissaient par milliers, la sanction tourne à la démonstration,

11. Origène, Contre Celse, trad. Marcel Borret, Paris, Le Cerf, 1969, VIII, 44.

12. On notera que les montanistes et les marcionites qui prétendaient au martyre furent systématiquement discrédités par l'Église. Celle-ci s'évertua à faire passer les hérétiques pour « suicidaires » en se servant des mêmes arguments dont les juges romains usaient à l'encontre des fidèles catholiques. Ainsi Clément d'Alexandrie, dans ses Stromates, proclame-t-il que « ceux qui se précipitent à la mort - car il en est quelques-uns, qui ne sont pas des nôtres, qui n'ont rien de commun avec nous que le nom, et qui, dans leur haine pour le Créateur ont de la hâte en se livrant à la mort : des assassins, ces misérables ! nous disons que ceux-là se font périr eux-mêmes sans rendre témoignage, bien qu'ils soient officiellement punis. » 
la repentance à l'apologie. La patience du martyr ne s'apparente ni par la détermination, ni par le renoncement à la constance du sage : c'est une scénographie ${ }^{13}$. Marc-Aurèle (121-180) ne dit pas autre chose. Il faut, explique-t-il, que «la préparation [à la mort] vienne de notre jugement propre, qu'elle ne réponde pas, comme chez les chrétiens, à la simple opiniâtreté, mais qu'elle soit réfléchie, sérieuse, et pour convaincre aussi les autres, qu'elle ne soit pas théâtrale. ${ }^{14}$ (Je souligne)

La théâtralisation du supplice induit dans la chaîne judiciaire un surcroît de représentation. À la contemplation vide de l'exécution capitale se substitue celle du corps martyrisé soumis aux sévices. Or qu'est-ce qu'un corps martyrisé ? Un corps qui non seulement décide de sa mort, mais du sens qu'elle va prendre. C'est un corps qui interprète sa mort. Acteur de sa passion, le martyr incarne au-delà de sa situation la passion du Christ, qu'il produit et rejoue. Certes le martyr n'est pas pour ainsi dire en représentation, mais il représente assurément sa conviction : c'est d'ailleurs tout le problème. L'individu, transcendé par la communauté dont il émane et dont il est le représentant, échappe à la justice qui ne condamne jamais en lui qu'un imitateur dont le modèle reste intouchable. Le tour de force consistant à commuer la condamnation en signe d'élection. Par le truchement du rôle qu'il incarne, le chrétien mené au martyre transforme le code de l'enquête pénale établie sur une culture de la preuve, en un témoignage magistral chargé d'administrer la preuve de la foi. Comment «après des preuves si importantes » rester incrédule? Comment « hésiter encore à se prononcer au sujet de la destruction de la mort et de sa fin »? Comment avoir « l'impudence de nier des faits aussi évidents. » ${ }^{15}$ ? Tels sont les muets reproches que le corps torturé adresse à la foule, lui qui doit convaincre, conforter et convertir à ses dépens. Il apparaît donc que la décision de justice, du moins en ce qui concerne sa signification et son application, se trouve

13. Voir mon étude, « La patience du martyr », Critique, n 658, 2002.

14. Marc-Aurèle, Pensées, trad. Amédée I. Trannoy, Paris, Les Belles Lettres, 1983, XI, 3.

15. Athanase d'Alexandrie, Sur l'incarnation, trad. Charles Kannengiesser, Paris, Le Cerf, 2000, 28, 4. 
entièrement résorbée, refondue dans le système de croyance de l'Église. L'Église exploite ses forces perdues grâce à la fécondité « médiatique » de cette déperdition : l'énergie dépensée accroît la puissance de l'Église. En effet, «l'opprobre de ceux qui souffrent persécution pour la justice, endurent toutes sortes de tourments, sont mis à morts $[\ldots]$ seule l'Église le supporte purement : sans cesse mutilée, sur-le-champ elle accroît ses membres et retrouve son intégrité. » ${ }^{16}$ En choisissant donc de valoriser le martyre, c'està-dire en créant une valeur, l'autorité religieuse parasite le pouvoir à la source même. Prenant à contre-pied la justice, elle recycle intégralement le résidu de l'opération pénale - le corps du condamné ${ }^{17}$ et l'injecte dans le circuit de son économie « médiatique ». De fait, l'Église n'invente pas une nouvelle position sociale, mais le discours qui transvalue cette position. Grâce à ce discours, elle transforme la justice en chaîne de production : au lieu d'éliminer le corps rebelle, l'appareil judiciaire va donc fabriquer pour l'Église un corps glorieux.

Exposée au plus grand nombre, l'esplanade des martyrs est un instrument de propagande puissant pour une Église en mal de publicité. Le tribunal, réaménagé en tribune, est le lieu où se publient les bans du contre-pouvoir. Mais la tribune qu'occupe le martyr, la scène sur laquelle il évolue, parce qu'elle échappe à la justice des hommes, n'en est pas moins strictement régentée et codifiée. L'inculpé qui s'avance vers ses juges a conscience que ses réponses sont déterminantes s'il veut entrer dans les rangs des « bienheureux ». Il veillera donc à conformer ses déclarations aux innombrables modèles auxquels on lui propose de s'identifier : le Christ, bien sûr, modèle par excellence, mais aussi certains évêques ou docteurs martyrisés au II siècle $^{\mathrm{e}}$ (Ignace, Justin, Polycarpe) qui, par leur conduite, devenaient de nouvelles figures de référence. Ainsi les témoins du supplice de Polycarpe ne manquent pas de souligner que l'évêque de Smyrne «a attendu d'être trahi, comme le Seigneur, pour nous apprendre à

16. Irénée de Lyon, Contre les hérésies : dénonciation et réfutation de la gnose au nom menteur, trad. Adelin Rousseau, Paris, Le Cerf, 2000, IV, 33, 9.

17. La justice de l'Empire tenait les exécutions secrètes et soustrayait le corps à la vue de tous. 
l'imiter, nous aussi. » ${ }^{18}$ À l'édification par l'exemple s'ajoutent encore les exhortations et les actes des martyrs qui, pour leur auditoire, avaient valeur de norme. Car ces lettres et ces récits offraient autant de lectures idéologiques des persécutions perpétrées, distribuant bons et mauvais points aux impétrants selon qu'ils abjurent ou non la foi. Consignés et validés par l'Église, ces cas édifiants mettent en scène les attitudes et les réponses que le martyr doit produire pour ne pas tromper les espoirs de sa communauté. Il ne faut donc pas oublier que la part de prosélytisme qui entre dans le spectacle du martyre est une part ajoutée, entièrement produite et fabriquée, une composante artificielle, et que le credo que professe le martyr, luimême, est une élaboration secondaire. Le martyr est produit par le tribunal mais contrôlé par l'Église. Condamné par le premier, il obéit aux instructions de la seconde et se voit le plus souvent dépossédé de toute initiative individuelle. « On ne devient un martyr, note MarieFrançoise Baslez, que parce que les autres ont fait de vous un martyr ${ }^{19}$. C'est pourquoi devant le juge, le martyr ne répond pas seulement de lui-même, mais pour l'Église entière. «La puissance de l'Esprit $»^{20}$ qui habite le martyr se voit ainsi commuée en pouvoir politique par l'intermédiaire du groupe qui assure sa transformation. Le groupe façonne à son image le corps du martyr afin qu'il le représente; et, dans la mesure où il le représente, celui-ci imite le modèle de sa foi. Ce n'est pas l'ardeur à confesser qui fait le martyr, mais l'institution. C'est l'Église qui évalue si la profession de foi est adéquate au système de croyance qu'elle représente, et qui tranche en dernière instance. La mainmise de l'autorité sur ses sujets est totale. Autant dire que l'Église répond non seulement pour le martyr, mais à sa place. Très concrètement, le martyr ne rend pas témoignage, il récite. Dans quelles proportions ? Cet extrait de correspondance entre l'évêque de Carthage (200-258) et une poignée de chrétiens emprisonnés le laisse imaginer : « Vos lettres, très cher Cyprien, nous

18. Ignace d'Antioche, « Polycarpe de Smyrne » dans Lettres, trad. Pierre Alfred Camelot, Paris, Le Cerf, 2006, I.

19. Marie-Françoise Baslez, Les persécutions dans l'Antiquité. Victimes, héros, martyrs, Paris, Fayard, 2007, p. 257.

20. Irénée de Lyon, Contre les hérésies..., V, 9, 2. 
ont toujours parlé, en l'adaptant aux circonstances, un langage plein de nobles sentiments, en les lisant assidûment ceux qui sont dans l'erreur se corrigent, les hommes de vraie foi se sentent affermis. En expliquant sans cesse dans vos écrits les mystères cachés, vous augmentez la foi en nous, et vous amenez les profanes à croire [...] Comme un vrai et bon maître vous avez dit devant le gouverneur ce que nous devions répondre; vous l'avez proclamé dans les actes pro-consulaires [...] Ceux qui sont condamnés avec nous vous adressent devant Dieu les plus grandes actions de grâces, très cher Cyprien, de ce que votre lettre a rendu vigueur aux cœurs. » ${ }^{21}$ (Je souligne)

Alors qui s'exprime ? Qui témoigne ? Qui parle ? Non le martyr, mais l'autorité canonique, elle seulement. Le pouvoir ecclésial canalise et surveille la puissance de son élite en conditionnant son discours et en limitant sa liberté d'expression. Les martyrs ne parlent pas. Ils sont interdits de parole et, lorsqu'ils rendent « témoignage », il n'est que l'expression des intérêts généraux. Le groupe, dont ils dépendent étroitement, se charge à leur place de toujours dire ce qu'il faut. Le chrétien martyrisé est un acteur, un imitateur capable de jouer un rôle dans lequel lui-même, son individualité, se dissout. Le corps martyrisé disparaît derrière le corps impersonnel de l'événement qu'il incarne. À la fois imitateur du modèle et modèle pour autrui, tout à la fois Christ, athlète et soldat, expression du dogme et attribut du groupe, il n'est lui-même personne. À la surface de sa peau, se scelle l'identité de la communauté. Mais son corps a définitivement cessé de lui appartenir. Gestes, postures, paroles, tout lui est prescrit, son rôle préparé, sa fonction élaborée. On lui dicte l'attitude à prendre, le jeu à produire, le propos qu'il convient de tenir, les exemples auxquels se conformer et les obligations qui incombent à son personnage. Bref, on le dirige.

Avec les privations, les contritions, les mortifications, les exactions volontairement subies, c'est donc la douleur qui organise sa propre représentation et ce spectacle, le spectacle de la souffrance, livre une image fidèle de la place qu'occupe à cette époque le corps sur la

21. Cyprien de Carthage, Correspondance, trad. Louis Bayard, Paris, Les Belles Lettres, 1961-1962 LXXVII, I-III. 
scène publique. Le fait de contenir sa peur et de maitriser sa douleur, qui passait jusqu'alors pour une marque de courage, de vaillance et de vertu, et qu'on manifestait traditionnellement sur le champ de bataille ou dans l'arène, sont à présent interprétées comme des signes de force et de puissance. Certes l'imaginaire du guerrier ou du gladiateur a bien essaimé dans la littérature chrétienne soit à titre de comparaison, soit à titre d'exemple, mais ces archétypes sont toujours appelés à être dépassés : « Si les seuls honneurs terrestres permettent de donner force au corps et à l'âme de manière à mépriser l'épée, le feu, la croix, les fauves et les tortures pour prix d'humaine reconnaissance, je peux affirmer que ces douleurs sont peu de choses en comparaison de l'honneur céleste et de la récompense divine. ${ }^{22}$ (c'est moi qui souligne). La force qu' accumule le corps en se privant des plaisirs du siècle et en supportant dans la joie les cruautés du bourreau, ne figure pas seulement l'œuvre de la volonté du martyr, sa détermination et sa vigueur, mais exhibe sous des dehors visibles la détermination de sa foi. L'ampleur du renoncement n'a d'égal que l'engagement dans la foi. Ce point mérite d'être souligné car, ce faisant, l'Église inscrit la foi dans un régime de visibilité inédit, qui n'aura de cesse d'être renforcé par la suite. D'un côté, la véritable piété passe par l'acceptation complète de son sort qui dépend de la providence divine, le pouvoir ecclésiastique cherchant à légitimer la disgrâce du martyr par le sens de sa foi. De l'autre, le martyr administre par sa disgrâce même la preuve de sa foi aux yeux de tous. Le paradoxe se laisse résumer ainsi : la foi légitime le martyr qui légitime la foi. Seulement, cette preuve ne s'adresse pas au jugement, au bon sens ou à la raison, mais à la vue. Le martyr est la preuve vivante - et surtout visible - de l'existence de Dieu. Si bien qu'il était plus fortifiant pour les croyants de se recueillir aux côtés du futur martyr, dans la prison, plutôt que dans la célébration posthume de ses exploits. Une attention toute particulière était alors portée à ces derniers par l'Église afin, comme on le verra, de contrôler les agissements de ces héros très populaires.

22. Tertullien, Ad martyras, IV, 9 (traduction personnelle). 


\section{L'AURA DES MARTYRS}

Il faut dire que les prisonniers jouissaient durant toute la durée de leur incarcération soit en prison, soit aux mines, d'un régime de faveur bien particulier en raison du soutien que leur apportait leur communauté, régime de faveur qui paraîtra d'autant plus surprenant au regard de leurs conditions de détention effectives. En évoquant le sort de ces hommes confinés dans des cellules collectives, les sources sont unanimes à mentionner le manque d'hygiène, l'insalubrité, les carences alimentaires, l'exposition au froid et à la chaleur, le dénuement vestimentaire, parfois la mobilité réduite, la mise aux fers et la bastonnade, la privation de sommeil et les humiliations corporelles. «Fondé sur l'arbitraire, la corruption et probablement la perversité, estime Yann Rivière, le traitement des détenus paraît s'écarter régulièrement des normes admises par la loi. » ${ }^{23}$ Loin du juge, le gardien règne en maître sur l'univers carcéral ; c'est lui qui accorde les autorisations de visite, qui tolère les offrandes de nourriture ou de vêtements, qui laisse entrer les païens pour convaincre les chrétiens d'apostasier ou qui, selon son humeur, remise ceux-ci au fond du cachot. Pourtant, malgré la somme d'épouvantes que représente la prison, ce lieu d'exclusion sociale est aussi le centre d'une activité intense pour la confrérie chrétienne. En effet, il n'est pas de gardien qui ne se soit laissé soudoyer, pas de porte qui ne se soit laissée franchir, pas de compagne ou de compagnon qui n'ait pu assister le captif dans sa détention, pas de vivres qui n'aient pu être apportés au fond de ces mitards. D'ailleurs, la visite aux prisonniers s'impose vite comme le modèle par excellence de la charité à la fraction de chrétiens qui n'était pas inquiétée par l'autorité romaine. L'Église elle-même favorisait et soutenait ces œuvres caritatives : « Vous ne devez pas avoir honte d'aller les trouver en prison ; car si vous le faites cela vous sera compté comme martyre; en effet, le martyre, eux, ils en font l'expérience, mais pour vous il est dans votre ardeur à vous associer à leur combat $»^{24}$. Finalement, tout se

23. Yann Rivière, Le cachot et les fers : Détention et coercition à Rome, Paris, Belin, 2004, p. 225.

24. Les Constitutions apostoliques, trad. Marcel Metzger, Paris, Le Cerf, 1992, 5, 1, 2-5. 
passe comme si le martyr, du fond de sa cellule, à proportion de la liberté qui lui est ôtée, concentrait sur lui un pouvoir dont l'influence continuait de s'étendre bien au-delà des limites matérielles de son lieu de détention. Cette nouvelle source de pouvoir, l'Église, malgré le désir qu'elle en avait, ne l'a pas toujours tenue sous contrôle. Parce que les martyrs étaient les vrais représentants de la sainteté et de la foi, les plus populaires et les plus visibles, ils entraient en concurrence directe avec la légitimité du pouvoir ecclésiastique si bien que celui-ci a toujours cherché à les maintenir sous sa coupe, voulant à tout prix éviter l'émancipation de leur voix qui menaçait de se faire dissidente.

Voilà pourquoi le cas de Pérégrinus ${ }^{25}$, ce pseudo-philosophe converti au christianisme dans le seul espoir de tirer bénéfice de la solidarité et du secours matériel de la communauté, plaide avec force pour un rapport problématique de l'autorité ecclésiastique avec ses dévoués sujets. Car ce dernier n'a pas hésité, dès lors que cela pouvait conférer à sa personne la célébrité, la puissance et la faveur publique qu'il convoitait, à se réfugier dans la chapelle du christianisme. Or, en infiltrant la congrégation chrétienne au moment où se met en place le réseau de solidarité entre frères de même croyance, s'immisçant dans l'univers fermé des prisons aux côtés de chrétiens convaincus et profitant à son usage de toutes les failles que recelait le système de soutien aux détenus élaboré par l'Église, Pérégrinus nous offre l'occasion de mettre à nu, sans éléments hagiographiques, les mécanismes de la fabrication du martyr. Je citerai longuement le texte de Lucien qui nous servira de guide pour la discussion de ce point :

Après avoir assassiné son père en l'étouffant, « l'affaire s'étant répandue, Pérégrinus s'enfuit, se condamne à un exil volontaire et se met à errer de pays en pays. Ce fut vers cette époque qu'il se fit instruire dans l'admirable religion des chrétiens, en s'affiliant en Palestine avec quelques-uns de leurs prêtres et de leurs scribes. Que vous dirai-je ? Cet homme leur fit bientôt voir qu'ils n'étaient que des enfants ; tour à tour prophète,

25. Cf. Lucien de Samosate, «La mort de Pérégrinus » dans Euvres compètes, trad. Eugène Talbot, Paris, Hachette, 1912. Pour une affaire similaire, voir le cas de Priscillien. Cf. Sulpice Sévère, Chroniques, trad. Ghislaine de Senneville-Grave, Paris, Le Cerf, 1999, 2, 51, 7. 
thiasarque, chef d'assemblée, il fut tout à lui seul, interprétant leurs livres, les expliquant, en composant de son propre fonds. Aussi nombre de gens le regardèrent-ils comme un dieu, un législateur, un pontife, égal à celui qui est honoré en Palestine, où il fut mis en croix pour avoir introduit ce nouveau culte chez les hommes. »

Je n'insiste pas sur ce premier passage, dont l'authenticité reste controversée, où Lucien donne libre cours à sa verve satirique. On ne peut en effet s'empêcher de croire que l'épisode de cette mystification se soit présenté à l'auteur comme l'occasion rêvée de se moquer de la superstition chrétienne : l'usurpateur avait quelque talent et son immersion réussie dans les sphères d'influence de l'Église décelait assez par elle-même ce qu'avait de crédule et d'opportuniste ce pouvoir prétendument spirituel. Réservons plutôt notre attention à la suite du récit :

« [Pérégrinus] ayant donc été arrêté par ce motif, fut jeté en prison. Mais cette persécution lui procura pour le reste de sa vie une grande autorité, et lui valut le bruit d'opérer des miracles et d'aimer la gloire, opinion qui flattait sa vanité. Du moment qu'il fut dans les fers, les chrétiens, se regardant comme frappés en lui, mirent tout en œuvre pour l'enlever ; mais ne pouvant y parvenir, ils lui rendirent au moins toutes sortes d'offices avec un zèle et un empressement infatigables. Dès le matin, on voyait déjà rangée autour de la prison une foule de vieilles femmes, de veuves et d'orphelins. Les principaux chefs de la secte passaient la nuit auprès de lui, après avoir corrompu les geôliers. Ils se faisaient apporter toutes sortes de mets, lisaient leurs livres saints ; et le vertueux Pérégrinus, il se nommait encore ainsi, était appelé par eux le nouveau Socrate.

Ce n'est pas tout ; plusieurs villes d'Asie lui envoyèrent des députés au nom des chrétiens, pour lui servir d'appuis, d'avocats et de consolateurs. On ne saurait croire leur empressement en de pareilles occurrences : pour tout dire, en un mot, rien ne leur coûte. Aussi Pérégrinus, sous le prétexte de sa prison, vit-il arriver de bonnes sommes d'argent et se fitil un gros revenu. Ces malheureux se figurent qu'ils sont immortels et qu'ils survivront éternellement. En conséquence, ils méprisent les supplices et se livrent volontairement à la mort. Leur premier législateur leur a encore persuadé qu'ils sont tous frères. Dès qu'ils ont une fois changé de culte, ils renoncent aux dieux des Grecs, et adorent le sophiste crucifié dont ils suivent les lois. Ils méprisent également tous les biens et les mettent en commun sur la foi complète qu'ils ont en ses paroles. En sorte que s'il vient à se présenter parmi eux un imposteur, un fourbe adroit, il n'a pas de peine à s'enrichir fort vite, en riant sous cape de leur simplicité. Cependant Pérégrinus est bientôt délivré des fers par le gouverneur de Syrie [...] qui savait notre cynique assez fou pour se 
livrer à la mort dans le dessein de s'illustrer. Il le fait mettre en liberté, ne le jugeant digne d'aucune punition. »

La figure du martyr qui récitait sa leçon devant le proconsul, sacrifiait sa personne sur l'autel du groupe et maîtrisait la signification de sa mort, cette silhouette semble tout à coup presque pâle et trop lisse au regard des agissements froidement prémédités de Pérégrinus. C'est qu'on avait peut-être trop vite enterré la part de jalousie et de convoitise, de vaine gloire qui se mêle à la valorisation du martyre sous l'image de l'homme docile et droit qui file doux vers le bûcher. Évidemment, l'opération du martyre est avant tout une opération rentable en termes de visibilité pour l'Église, mais c'est encore, ce qu'on dit rarement, une fabrique de centres hétérogènes, de lieux ouverts à tous les commerces et à toutes les spoliations, zones indistinctes où se coudoient autorité, débauche et richesse. Partout où se concentre la puissance, il y a prolifération des pouvoirs. La position enviable qu'avait créée l'Église pour ses fidèles, les plus roués d'entre ceux-là se l'approprient pour leur avantage personnel. Apollonius (mort en 185) dénonce ainsi les pratiques de Thémison «qui a revêtu sa cupidité de dehors avantageux et qui, n'ayant pu porter le signe de la confession, a déposé les fers au moyen d'une grande somme d'argent. Pour cela même il aurait dû s'humilier ; mais il a osé se glorifier comme martyr, et il a singé l'apôtre, et, composant une lettre catholique, catéchiser les gens qui ont une foi meilleure que la sienne. ${ }^{26}$ Les bénéfices matériels, le prestige social procurés par la condition chrétienne poussent les meilleurs fidèles à toutes les audaces jusque dans l'isolement des fers. De fait, les antichambres des cachots qui recèlent les candidats au martyre sont la proie d'une effervescence sans pareille ; Cyprien ne cesse d'exhorter prêtres et confesseurs en visite dans les prisons à plus de retenue : ceux-ci devraient s'abstenir « de coucher dans les locaux où couchent des femmes », d'avoir «des relations viles et infamantes » avec elles ou encore de se «montrer en état d'ivresse » ${ }^{27}$. C'est que la prison est un lieu d'inconduite et de collusion. On s'y retrouve, on

26. Eusèbe de Césarée, Histoire ecclésiastique, trad. Gustave Bardy, Paris, Le Cerf, 2001, 5, 18, 5-7.

27. Cyprien de Carthage, Correspondance..., XIII, IV, 1-5. 
s'y réunit et, à la faveur de la clandestinité, on y passe des arrangements sans aucune conformité avec la position officielle de l'Église : le cœur des prisons est hors contrôle, bien dissemblable de la sage image d'Épinal qu'en donnent les apologistes. Car c'est toute une foule interlope, avide de pouvoir et servile, qui transite par la cellule du martyr à la recherche de l'impunité que lui conférera la bénédiction du prisonnier. À l'incessant trafic des visiteurs externes qui, chargés de vivres et d'argent, viennent soulager la détention du condamné avec l'espoir d'obtenir de sa part l'absolution, répond le long cortège des prêtres et des confesseurs aux « mœurs perverses ${ }^{2} 8$ qui, loin de « suggérer [aux martyrs] de se conformer aux préceptes divins, les en empêchent plutôt. » ${ }^{29}$ La renommée et le crédit acquis par tous les détenus chrétiens étaient tels qu'on les croyait capables de réaliser des miracles, de guérir les maladies et d'accorder la vie éternelle en vertu du chemin de croix qu'ils accomplissaient. Pour accomplir le devoir de charité, et sans doute aussi par admiration, « beaucoup se sont livrés aux fers afin d'en racheter d'autres; beaucoup se sont livrés comme esclaves et avec le prix de la vente ont donné de la nourriture à d'autres ; beaucoup de femmes, rendues fortes par la grâce de Dieu, ont accompli bien des actions viriles $»^{30}$. Si bien qu' autour du pôle d'affluence que représente le martyr dans les fers, c'est tout une économie parallèle qui s'installe et se développe, basée sur l'achat et la vente illicites d'indulgences ${ }^{31}$. Illicites, parce que le martyr, sans tenir compte des critères sélectifs de l'Église, ni de sa liturgie, bénit, sanctifie, soigne, intercède, remet les péchés et délivre des places pour le royaume des cieux à ceux qui viennent le trouver. Il ne faut pas chercher à minimiser la proportion du phénomène car, à éplucher la correspondance de Cyprien, on se rend à l'évidence que ce trafic n'avait rien de marginal, ni dans son ampleur, ni dans son rendement, et qu'en cette deuxième moitié du

28. Id., op. cit., XIV, III, 2.

29. Id., op. cit., XV, I, 1.

30. Clément de Rome, Épîtres aux Corinthiens..., LIV, 2-3.

31. Il faut à tout prix, dit Cyprien, « réprimer ceux qui font acception de personne dans la distribution des bienfaits et cherchent l'occasion d'une complaisance ou d'un trafic illicite. » (je souligne). 
IIII siècle, l'autorité ecclésiastique était absolument incapable d'endiguer son expansion ou même seulement de la contenir. Ce commerce constituait, en réalité, une menace latente pour l'unité de celle-ci. L'inconduite des prêtres et des confesseurs, relevée et condamnée par l'évêque de Carthage, est à mettre au même plan que celle de leurs homologues dévolus aux supplices : elle cause du tort à l'Église et sa vitrine, les martyrs. Ceux qui, pour jouir d'un respect et d'une renommée populaire, absolvent en masse les lapsi ne respectent évidemment pas les consignes de leur hiérarchie et faillissent à toutes leurs obligations morales : "Quel péril, en effet, ne devons-nous pas redouter de la colère de Dieu, quand quelques prêtres ne se souvenant ni de l'Évangile, ni de leur dignité, ne songeant pas davantage ni au jugement à venir du Seigneur, ni à l'évêque qui est actuellement leur chef, osent, ce qui ne s'est jamais vu sous nos prédécesseurs, outrager et mépriser leur chef en s'arrogeant tous les droits [...] Ceux-là en cherchant à être populaires, sans viser à rendre la santé spirituelle, font plutôt du tort aux lapsi. [...] ils risquent ensuite de rendre impopulaires les bienheureux martyrs [... en offrant] pour eux le sacrifice et leur donn[a]nt l'Eucharistie. ${ }^{32}$

Du martyr qui signe le libelle au prêtre qui l'exploite, il n'y a qu'une seule et même chaine d'action qui, pour le plus grand bénéfice de ceux qui y participent, s'accroche à la position avantageuse du martyr. Partout c'est la même insubordination, la même recherche de profit qui se rencontre. Un véritable réseau semble avoir fonctionné avec le libelle pour monnaie et l'indulgence comme perspective de gain. Mais face à la montée en puissance des lapsi qui, sur la foi des billets qu'ils ont obtenus des condamnés, réclament leur dû, il n'est pas d'autorité assez forte qui puisse enrayer ce mouvement d'indiscipline. Dans cette course au profit où chacun tente de tirer sa part du jeu, les conservateurs de la foi sont débordés par la vigueur du mouvement, par les réclamations de cette foule qui, contrairement aux calculs les plus pessimistes des autorités, tient sa légitimité de la part la plus légitime de l'Église. De toute évidence, la nouveauté du problème jette la confusion dans les rangs du clergé qui cherche

32. Cyprien de Carthage, Correspondance..., XVI, I, 2. 
une parade pour « tout à la fois rendre honneur aux martyrs et arrêter ceux qui voulaient mettre le désordre partout $»^{33}$. Car c'est bien de désordre dont il s'agit, et même de « véritable sédition » ${ }^{34}$ : les autorités sont prises à partie, leurs représentants interpellés, malmenés voire molestés ; on intimide, on crie pour éprouver la résistance de l'interlocuteur, on le presse pour obtenir la validation d'un libelle chèrement acquis : « Ce désordre a déjà commencé à se manifester. Dans un certain nombre de villes de notre province, les lapsi se sont portés en foule vers les dépositaires de l'autorité et les ont forcés à leur donner, sur-le-champ, la paix qu'ils criaient leur avoir été accordée à tous par les martyrs et les confesseurs. Usant ainsi d'intimidation, ils ont forcé la main à des chefs qui n'avaient pas assez de force d'âme ou de vigueur de foi pour résister. » ${ }^{35}$ Les martyrs eux-mêmes, malgré leur possibilité d'action réduite liée à leur enfermement, malgré leur isolement régulièrement requis par la justice impériale, malgré la diminution de leur état physique et moral, semblent toujours faire front commun. Et si l'on songe à Pérégrinus, on se rappellera qu'il avait dû se fondre dans le grand réseau social de la prison pour tirer tout le bénéfice de sa claustration. Qu'ils agissent au nom de leur intérêt personnel, à l'image de Pérégrinus, ou en vue de l'intérêt collectif, les futurs martyrs, « faisant peu attention à l'Écriture dans l'ivresse de leur triomphe » ${ }^{36}$ et acceptant de signer des libelles pour des motifs qui vont du népotisme à la subornation, contreviennent à la politique épiscopale, la court-circuitent par leurs initiatives et la remettent en question dans sa globalité : c'est là le point essentiel. Le rapport de force entre sujets du Christ et représentants de l'Église est en train de se modifier profondément, à la défaveur des officiels. La question qui se pose à eux n'est maintenant plus tellement de savoir comment le martyr doit représenter l'Église, mais comment empêcher qu'il relègue ses décideurs à l'arrière-plan du débat résurrectionniste. Entre Dieu et le martyr, qui sont en relation directe, l'évêque apparaît dans une

33. Id., op. cit., XX, III, 2-3.

34. Marie-Françoise Baslez, Les persécutions..., p. 325.

35. Id., op. cit., XVII, III, 1.

36. Id., op. cit., XVII, III, 1. 
position surnuméraire. Et, devant la foule qui se présente aux portes des centres religieux munis de leurs libelles, son influence semble mince en comparaison de celle du martyr.

\section{L'INVENTION DES RELIQUES}

Face au paradoxe chrétien, la stratégie des autorités romaines se modifie. Dès le $\mathrm{III}^{\mathrm{e}}$ siècle en effet, le régime des exécutions passe d'une cadence lente où la sanction, exemplaire, garde encore une portée édifiante à un rythme soutenu de répression massive pratiquant la politique de la terre brûlée : « tous d'un commun accord, consigne Eusèbe (265-340), se précipitèrent sur les maisons des fidèles, et tombant chacun sur ceux qu'il connaissait, les voisins, ils les emmenèrent, les volèrent et les pillèrent. Les objets les plus précieux de leurs trésors étaient dérobés; les objets sans grandes valeurs et ceux qui étaient faits en bois étaient jetés et brûlés sur les chemins, de manière à donner le spectacle d'une ville prise par les ennemis $»^{37}$. L'intensification des représailles en guise de réplique sanglante à l'obstination des sectateurs signe un renouveau dans l'interprétation de l'échiquier politique. Mais, parce que ce revirement stratégique augmentait sensiblement le chiffre des prévenus dans les lieux de détention, il a aussi contribué à accélérer l'évolution des mœurs carcérales. Tandis que la cible de l'appareil répressif impérial se déplaçait, la population des prisons romaines, elle, changeait de visage. Désormais on n'arrête plus seulement les endoctrinés qui, tout en maintenant qu'ils sont chrétiens, refusent de sacrifier aux images de l'empereur et des dieux, mais tous les garants de l'autorité chrétienne de manière systématique. Non seulement les chefs locaux, «évêques, prêtres ou diacres ${ }^{38}$, mais encore les personnalités influentes du monde romain qui auraient confessé le Christ.

Durant les années qui suivent le déclenchement des grandes persécutions, le nombre des prisonniers arrêtés pour délit d'athéisme

37. Eusèbe de Césarée, Histoire ecclésiastique, trad. Gustave Bardy, Paris, Le Cerf, 2001, VI, 41-42.

38. Cyprien de Carthage, Correspondance..., LXXX, 1. 
va sensiblement enfler. Ces hommes, lettrés pour la plupart, théologiens aguerris, orateurs frottés de dialectique, viennent grossir les rangs de la foule des petits crédules, attentistes, dévots et autres illuminés. L'ère industrielle de la persécution antichrétienne se caractérise donc autant par l'augmentation des arrestations que par la diversité des catégories sociales que brassent les centres d'incarcération. Qu'est-ce que cela implique ? Cette foule hétéroclite qui compose la faune des culs-de-basse-fosse de l'Empire, clairsemée de personnalités charismatiques, malgré son caractère hétérogène fortement marqué, s'identifie comme un seul corps. Et la foule non moins grande de leurs admirateurs solidaires ne les identifie pas moins comme une communauté. C'est que la population composite des cachots s'était organisée selon des lignes d'intelligences qui lui étaient propres et avec des moyens qui, bien que rudimentaires, démontreront leur efficacité. La circulation de biens et de marchandises qui transite entre l'intérieur et l'extérieur de la prison n'est qu'un aspect manifeste, patent, de la chaîne de communication développée autour du pouvoir d'absolution des martyrs. La politisation des populations incarcérées, l'organisation de réseaux au sein de la prison montrent combien l'aura médiatique dégagée par les martyrs a su être exploitée au maximum par l'intermédiaire du système de distribution d'à-valoir.

L'influence de ce pôle aura contribué pour une part difficile à quantifier à ce qu'émerge dans une société romaine traditionnellement soucieuse du respect envers les morts ${ }^{39}$ un culte pour les reliques. Car le goût des reliques n'est pas inhérent au christianisme. Soustraire, diviser, segmenter, parcelliser et thésauriser de la chair

39. Parmi tant d'autres, cet exemple tiré du Digeste (Paul), XLVII, 12, 11 : «Quant à l'action des profanateurs de tombeaux, s'ils extraient les corps eux-mêmes ou déterrent les os, on punira les pauvres (humiliores) à la peine maximale, on déportera les riches (honestiores) sur une île, mais on bannira les autres ou on les condamnera aux mines. ». Le respect du repos des défunts était partagé par la communauté des chrétiens ainsi qu'en témoigne cette épigraphe : «Ici, dans l'enceinte du martyr, le bienheureux Pasteur a fait construire/De son vivant un lieu de mémoire, il a lui-même achevé le tout,/Et l'a sanctifié. Qui veut violer ce lieu / Sait qu'il sera abattu. Cette peine lui sera réservée. » dans Anthologia latina, rec. F. Buecheler, M. Hakkert, Amsterdam, 1972, 1415. (Traduction personnelle). 
morte pour l'adorer n'a rien d'un geste ordinaire ${ }^{40}$, encore moins spontané. Cette pratique demeura problématique pour les plus conservateurs longtemps après la naissance d'un culte rendu aux restes des martyrs. Ainsi faut-il comprendre l'interpellation de Julien l'Apostat sur les mœurs des chrétiens : "Vous avez tout rempli de tombeaux et de sépulcres. Pourtant il n'a été spécifié nulle part dans vos Écritures qu'il fallait se vautrer dans les tombeaux et qu'il fallait les honorer. $»^{41}$ Il fallait donc pour outrepasser tous les tabous relatifs au cadavre, à l'impureté des morts et à la crainte de l'au-delà des raisons particulières que la ferveur populaire n'explique pas puisque, rien, dans la littérature vétéro- et néotestamentaire n'encourageait, ni n'autorisait l'apparition de ce goût. La haine du visible jointe au mépris de la matière semblait l'interdire au contraire pour longtemps. Car l'aniconisme chrétien, qui prévalait durant les premiers siècles de l'Église, s'honorait d'adorer son Dieu sans intermédiaire. On se rappelle par exemple que, lors de la tenue des procès contre les chrétiens, l'épreuve qualifiante consiste à sacrifier aux images du culte officiel : il fallait que «tous sans exception, hommes, femmes, serviteurs, même les enfants à la mamelle sacrifiassent, fissent des libations et goûtassent réellement des viandes consacrées par les sacrifices $»^{42}$. Le verdict de condamnation prenait, quant à lui, la forme suivante : «Que ceux qui n'ont pas voulu sacrifier aux dieux et obéir aux ordres soient fouettés et emmenés pour subir la peine capitale, conformément aux lois ${ }^{43}$. Ce point d'honneur fondé sur le rejet des vanités visibles possède une réalité concrète aussi bien que spirituelle pour le croyant qui ne veut pas se compromettre avec

40. Jack Goody et John Wortley sont à ma connaissance les seuls experts à attirer l'attention sur ce fait. Cf. Jack Goody, La peur des représentations : ambivalence à l'égard du théâtre, de la fiction, des reliques et de la sexualité, Paris, La Découverte, 2003 ; John Wortley, « The origins of Christian veneration of body-parts » dans Revue de l'histoire des religions, 1/2006.

41. Julien 1'Apostat, Contre les Galiléens, trad. Christopher Gérard, Bruxelles, Ousia, 1995, 335C.

42. Eusèbe de Césarée, Les martyrs en Palestine, trad. Gustave Bardy, Paris, Le Cerf, 1984, IX, 2.

43. «Justin » dans Les Actes des martyrs. Les premiers martyrs de l'Église \& Les martyrs de la grande persécution, trad. A. G. Hamman, Paris, Desclée de Brouwer, 1979, p. 40. 
les mœurs de son temps. La croyance en un Dieu unique privilégie l'exclusivité de ce rapport : il n'est pas d'autre culte qui puisse se soutenir en face du vrai culte d'amour que le fidèle doit à son protecteur. C'est pourquoi, lors de son martyre, en 185, Apollonius peut s'exprimer de la sorte : « Je ne puis honorer les idoles faites de mains d'homme. Aussi n'adorerai-je jamais or, ni argent, bronze ni fer, pas plus que de prétendues divinités de bois ou de pierre, qui ne peuvent ni voir ni entendre, mais sont l'œuvre d'ouvriers, d'orfèvres, de tourneurs ou de ciseleurs et qui n'ont pas de vie. ${ }^{44}$ L'argument d'Apollonius n'a pas encore atteint le degré de sophistication des rhétoriques byzantines se disputant le droit de figurer ou non le Christ. C'est en cela justement qu'il m'intéresse : il ne consiste qu'en la transposition d'un passage de l'Exode adapté aux circonstances contingentes du martyre. Cette adaptation est littérale et c'est ce qui importe pour mon propos car si, pour Apollonius, le texte sacré donne à lire la vérité littérale du monde, il va de soi qu'il en respecte les préceptes à la lettre. Pas question, donc, d'adorer la matière. C'est une évidence pour Apollonius, mais c'est également le moyen pour lui de marquer son identité chrétienne. Une posture conforme à son modèle de croyance qui, par sa spécificité, marque une différence de valeur. Dans une première phase, le rejet massif des valeurs romaines et des objets par lesquelles cette société s'identifiait s'est donc pensé comme un moment positif de la constitution de la communauté des croyants, une attitude de ralliement exactement au même titre que le mépris ostentatoire des conventions que pratiquaient les Cyniques. Ce rejet bannissait également tout espoir de survie terrestre qui prendrait la forme d'un culte, héroïsation ou divinisation, de même que les plus simples manifestations de recueillement dévolues à la commémoration. Cette indifférence à l'égard du devenir du corps après la mort, Ignace d'Antioche devait la rappeler à l'occasion de son exécution (entre 110 et 130$)^{45}$.

44. «Apollonius » dans Les Actes des martyrs..., p. 65.

45. Ignace d'Antioche, Lettres, trad. Pierre Alfred Camelot, Paris, Le Cerf, 2006, IV. La position d'Ignace reflète, semble-t-il, le sentiment général au début du deuxième siècle, car le martyre de Polycarpe (160) qui attesterait d'une vénération précoce des reliques anticipe, comme le souligne Marie-Françoise Baslez, « au prix de quelques anachronismes l'institution de ce culte ». 
Dans une configuration aussi défavorable au départ à l'apparition d'un culte des reliques, où les éléments de doctrine, les facteurs d'appartenance, tout comme le milieu social militent en faveur de son inexistence, comment comprendre, si on récuse le modèle de la "génération spontanée », l'émergence de cette pulsion morbide? Il y a dans cette apparition comme la trace d'un symptôme que les variables contextuelles ne permettent pas d'élucider. Son émergence correspond à une fonction, latente sans doute, enfouie mais qui n'est pas le rôle rassurant, légitimant auquel l'Église l'a assignée par la suite. Car il faut distinguer le plan de la justification, temps de l'élaboration secondaire, de la mise en récit et de l'explication, du plan de la formation, temps du surgissement, du passage à l'acte, de la rupture. Il faut dissocier le contenu de l'affect. On ne dira rien de la relique, de sa nature et de sa fonction, aussi longtemps qu' on reste sur le plan de la justification pour l'analyser. Lorsqu' on évoque les reliques en termes de «métonymie », «d'objet de mémoire », de « participation » ou de « sainteté », on ne fait jamais rien que reprendre les concepts opératoires que, dès le $\mathrm{IV}^{\mathrm{e}}$ siècle, les meilleurs théologiens développaient déjà. On restitue le discours dominant de l'Église qui, en justifiant a posteriori la pratique de cet usage, s'est imposé comme l'évidence qu'il n'était pas. Pour que la relique prenne la fonction d'objet de mémoire dans le code chrétien, il aura fallu l'y insérer en tant que signe dans la chaîne signifiante du discours théologique. Mais promouvoir la relique au statut de signe n'est pas une opération de discours. C'est une opération politique.

L'Église n'a délivré aucune contre-indication au développement du goût pour les reliques ; elle ne s'est pas non plus employée à le refréner. Nulle condamnation, nulle exhortation, nul rappel à l'ordre. Ce laisser-faire, recadré dans la politique de contrôle restrictive qu'exerce l'autorité ecclésiastique sur ses sujets, me paraît significatif ; ce n'étaient ni les justifications, ni les moyens d'agir qui faisaient défaut. Disons que pour tolérer et rendre acceptable une pratique aussi subversive au sein de sa société policée, l'Église devait y trouver avantage. En fait, il m'apparaît assez clairement que la dévotion pour le corps des saints prend naissance avec les exactions, multiples et réitérées, que commettent les Romains sur les cadavres 
de chrétiens. Ceux-ci étaient couramment mutilés, humiliés, voire privés de sépulture, contrairement aux dispositions légales en vigueur sur ce point. La jurisprudence romaine prévoyait en effet qu'à l'issue de l'exécution " les corps de ceux qui ont été condamnés à mort ne doivent pas être refusés à leurs parents ; [...] Les corps de ceux qui ont été condamnés au bûcher peuvent également être réclamés, afin bien entendu que les os et les cendres réunis puissent être transférés. ${ }^{46}$ Et cette loi s'appliquait aussi aux athées. Mais, par un surcroît de zèle ou de haine, les bourreaux refusaient souvent d'opérer la restitution parce que cet ultime outrage, comme nous l'apprend le récit des martyrs de Lyon (177), devait aider à extraire des esprits crédules toute croyance en la réincarnation : « On jeta à la curée le reste des confesseurs, étouffés dans la prison; nuit et jour, on montait la garde pour nous empêcher de les ensevelir. On exposa même ce que feu et fauves avaient épargné, des lambeaux de chair, des membres carbonisés. De ceux qui furent décapités, on laissa sans sépulture les têtes et les corps tronqués, sous la garde de soldats pendant de longs jours [...] nous ressentions cependant une grande peine de ne pouvoir confier leurs corps à la terre. Nous ne pouvions pas profiter de la nuit, ni séduire les gardes à prix d'argent ou par nos prières. Ils prenaient toutes leurs précautions, comme s'ils avaient grand intérêt à les laisser sans sépulture. Les corps des martyrs subirent tous les outrages et demeurèrent exposés pendant six jours. Ils furent ensuite brûlés et réduits en cendres que les scélérats jetèrent dans le Rhône, qui coule près de là, pour effacer jusqu'à leur trace sur terre. Les païens croyaient triompher de Dieu et priver les martyrs de la résurrection. "Il faut, disaient-ils, enlever à ces hommes jusqu'à l'espoir de la résurrection. À cause de cette croyance, ils introduisent chez nous une religion nouvelle et étrangère, méprisent les tortures et courent joyeusement à la mort". ${ }^{47}$

Le devoir de sépulture ainsi bafoué au mépris de la loi, on comprend mieux l'opiniâtreté que mirent les chrétiens à obtenir les dépouilles mortelles de leurs coreligionnaires. Mais la frontière qui sépare la compilation des restes du défunt en vue de les mettre en

46. Ulpien, Digeste, XLVIII, 23, 1 (traduction personnelle).

47. «Les martyrs de Lyon» dans Les Actes des martyrs..., p. 58. 
terre de la dévotion honorifique rendue à ces mêmes restes est mince. Dans les narrations hagiographiques, d'ailleurs, il n'est pas toujours aisé de démêler l'un de l'autre, encore moins d'interpréter la nature des gestes qu'accomplissent les fidèles. La confusion semble possible à tout instant. L'incertitude, totale, qui plane sur le sort du cadavre s'explique largement par le fait qu'il n'y avait justement pas de code établi pour diriger les conduites. En cette période où l'Église ne donne pas de consignes concernant l'ensevelissement de ses martyrs, la manœuvre de récupération des corps n'est dictée que par l'instinct des protagonistes et leur sens du devoir. Il arrive alors qu'on ne résiste pas à la sombre tentation de soustraire les restes encore fumants de la victime pour les dérober; peut-être pour leur rendre les derniers honneurs d'une manière plus intime ou personnelle. Mais ces ombres qui hantent les lieux d'exécution à la recherche des os et des cendres du martyr, quand ils les volent, reviennent vite à une attitude plus adéquate : «A la nuit tombante, [les fidèles] se rendirent en hâte à l'amphithéâtre. Ils emportaient du vin pour éteindre les ossements à demi brûlés. Cela fait, ils ramassèrent les reliques des martyrs et chacun en emportait pour son propre compte ce qu'il pouvait en prendre [...] mais il arriva qu'après sa mort, Fructosus apparût aux frères et les avertit de restituer sans retard ce que chacun par dévotion avait emporté de ses cendres, afin qu'elles fussent rassemblées en un même lieu. ${ }^{48}$

La relique est donc indissociable de l'élément funéraire lui-même. Elle accompagne le processus de deuil et se confond avec lui lorsqu'il s'agit d'aller enterrer les morts. Ainsi le fait que les reliques $e x$ contactu soient apparues sur le marché des « objets de mémoire » après les reliques ex corpore, et au bénéfice de l'effort d'élaboration déployé par les théologiens, tend à accentuer le rapport, étroit et premier, entre la volonté de donner sépulture à des corps en reste et celle, ambiguë, de rendre honneur aux restes mêmes. Naturels, ces agissements l'étaient aux yeux de ces fidèles qui, pour enlever les corps, soudoyaient leurs gardiens. Inscrits dans la lignée des soins dus aux morts, ils ne s'éloignaient pas des gestes cérémoniels ordinaires.

48. «Fructosus » dans Les Actes des martyrs..., p. 143. 
En 258, Cyprien, évêque de Carthage, subit son martyre. Il est respecté, populaire, admiré de son vivant. Sachant sa fin imminente, il ordonne à ses gens « de compter vingt-cinq pièces d'or » au bourreau afin de corrompre les gardes. Par cette requête, Cyprien montre qu'il souhaite être enseveli, reposer en un lieu consacré, mais sait-on s'il a conscience que sa sépulture sera vénérée ? Quand, spontanément, ses compagnons « étendent des toiles de lin et des serviettes » pour recueillir le sang du martyr, Cyprien ne le désapprouve pas. Lorsque ses frères enlèvent sa dépouille, leur responsabilité consiste d'abord à soustraire celle-ci à « la curiosité des païens » : il leur importe de préserver la dignité du mort des outrages de la foule. Ce n'est que la nuit venue que le corps est enlevé «à la lueur des cierges et des torches au milieu de prière ${ }^{49}$ pour être déposé en sa dernière demeure. Où s'arrête dans ce récit l'élément funéraire, où commence l'élément religieux ? Encore une fois, la frontière est ténue. On est en droit de penser que, pour ces hommes soucieux d'accomplir leur devoir, elle ne l'était pas moins. Constatons seulement que l'évêque vit sa passion sans partager l'émoi de ses frères, qu'il reste en retrait, passif, indifférent peut-être à son devenir terrestre. Ainsi durant toute la deuxième moitié du deuxième siècle et la première moitié du siècle suivant, l'Église a laissé ses fidèles libres d'inventer les gestes de dévotion qui, se répétant, allaient se ritualiser. Elle a permis le développement d'attitudes auxquelles, rétrospectivement, elle donnerait un fondement catholique.

Nous sommes trop renseignés sur l'agitation qui régnait dans les prisons de l'Empire au moment où se développe le goût pour les reliques pour ne pas être tentés de faire le rapprochement. Ainsi j'émets l'hypothèse que les reliques se sont présentées à un moment donné de l'Histoire comme une alternative avantageuse à la diffusion du message chrétien par les martyrs. Pourquoi avantageuse ? Parce qu'en des temps où la stabilité de l'Église était menacée par le nombre croissant de ceux qui «tombaient», les lapsi, les reliques avaient le pouvoir plus efficacement que ne l'auraient pu confesseurs et martyrs de ramener ces derniers dans le droit chemin. En se

49. « Cyprien» dans Les Actes des martyrs..., p. 120. 
montrant trop libéraux dans leur attribution d'indulgences, les martyrs avaient mis en péril l'unité de l'Église : comment allait-elle gérer les voix émancipées des martyrs ? comment allait-elle faire entrer dans le rang leurs « divergences d'attitude et de doctrine » ${ }^{50}$ ? comment ne pas se laisser gagner par la division ? La remarquable audience qu'avaient acquise les martyrs avait pris la hiérarchie ecclésiastique à revers ; les effets indésirables de leur succès n'avaient pas été calculés. C'est pourquoi l'Église se devait de réagir en désamorçant cette crise latente. En ce sens, le goût des reliques permettait à un moment opportun de fixer l'attention des croyants sur un médium neutre, signe vide qui ne demandait qu'à faire sens, qu'à remplir une fonction au sein de la chaîne signifiante religieuse. Neutre, la relique l'était, dès lors que sa récupération politique élevait ce médium au rang de signifié en refoulant le contenu morbide de cet élément. L'Église ne pouvait alors que s'intéresser à l'acquisition de ce médium dans la mesure où, en termes médiatiques justement, il se montrait plus efficace que la voix de ses martyrs. En orientant l'honneur dû aux martyrs sur son versant commémoratif, l'Église jouait donc la sécurité contre le risque de dissidence. Au fond, c'est parce que la voix de ses champions de la foi commençait à devenir difficile à contrôler, sinon impossible, que le pouvoir ecclésiastique s'est emparé de ce médium, hautement fiable, afin de favoriser sa politique d'implantation et d'expansion. Fabriqué sur mesure aux normes et aux besoins de l'Église, justifié selon sa science, cet outil de communication allait remplacer avantageusement, et pour longtemps, le témoignage de vive voix, source d'erreurs, de divergence, voire de dissidence. En inventant à cet objet une fonction dans la liturgie du culte, en lui trouvant une vocation, l'Église fixait sur un support vidé de toutes connotations mortifères l'attention des croyants, support dont elle pouvait gérer d'un bout à l'autre de la chaîne la répartition et l'utilisation. La relique présente l'avantage, une fois introduite dans la grammaire religieuse, d'être malléable au gré des volontés politiques qu'elle sert. Cette opération de calibrage, qui a consisté à accueillir dans son système un élément extrinsèque

50. Marie-Françoise Baslez, Les persécutions..., p. 325. 
pour l'intégrer à son code, ne s'est pas faite à grand renfort de publicité mais à l'ombre de quelques grands esprits affinant les notions de « participation » et de « grâce ». Car en pensant, à la suite de Platon, l'homme sur le modèle du réceptacle, les théologiens frayaient déjà la voie à ce que serait une pensée de la relique, écrin de la grâce descendue sur le martyr, et n'attendaient que le moment de se rendre maîtres de cet instrument de contrôle, capable de soumettre les consciences des croyants qui, de son pouvoir, attendent miracles et guérisons. En évacuant la part mortifère de la relique pour transformer cet élément somatique en vase de sainteté, en symbole de la foi, ils le faisaient entrer dans un régime de justification idéalisé, puisque sans signifiant. Ce changement d'état du médium, qui tout à coup d'ordure devient or pur ${ }^{51}$, renversait aussi bien les logiques d'appartenance que les signes de reconnaissance déployés par celles-ci, ouvrant la porte du christianisme aux objets de vénération de toutes sortes. Là où on rejetait comme signe de ralliement communautaire les images aussi bien que la matière, on allait désormais pouvoir, parce que le médium avait changé de régime de justification, adorer les images aux mêmes conditions que les reliques ${ }^{52}$.

bernard.bourrit@iil.ch

51. Cf. Ignace d'Antioche, «Polycarpe de Smyrne » dans Lettres... Après que son cadavre a été brûlé, les fidèles purent « enlever les ossements de Polycarpe [qui étaient] plus précieux que des gemmes, plus éprouvés que l'or le plus pur» (je souligne).

52. Je remercie M. Jean-Michel Roessli (Université de Sudbury, Canada) pour ses précieuses remarques. 